\title{
Exploring the Behavior of the Player to Change the Course of a Game
}

João Sousa

ISEL - Instituto Superior de

Engenharia de Lisboa/IPL

Rua Conselheiro Emídio Navarro

1959-007 Lisboa, Portugal

joaopimentasousa@gmail.com

Rui Jesus

NOVA LINCS,

Faculdade de Ciências e Tecnologia

Universidade Nova de Lisboa,

Quinta da Torre, 2829-516

Caparica, Portugal

ADEETC - ISEL/IPL

Rua Conselheiro Emídio Navarro

1959-007 Lisboa, Portugal

rjesus@deetc.isel.ipl.pt

Permission to make digital or hard copies of part or all of this work for personal or classroom use is granted without fee provided that copies are not made or distributed for profit or commercial advantage and that copies bear this notice and the full citation on the first page. Copyrights for third party components of this work must be honored. For all other uses, contact the Owner/Author.

Copyright is held by the owner/author(s).

CHI PLAY'16 Extended Abstracts, October 16-19, 2016, Austin, TX, USA ACM 978-1-4503-4458-6/16/10.

http://dx.doi.org/10.1145/2968120.2987737

\begin{abstract}
This paper proposes and discusses a horse racing game which explores the user behavior when playing, to change the gameplay. The game includes a module to capture and analyze, in real time, the body movement and the audio produced by the player. The paper describes the rational and design principles for the game and presents the main issues of the study conducted during the development of the game.
\end{abstract}

\section{Author Keywords}

Multimodal interfaces; Web games; Affective gameplay

\section{ACM Classification Keywords}

H.5.m. Information interfaces and presentation (e.g., $\mathrm{HCI}$ ): Miscellaneous;

\section{Introduction}

Watching a live sporting event in a stadium is one of occasions where people usually express emotions [3]. Some people are more reserved and can better control how they express these emotions but there are others that scream, shake their arms or even jump. When we are playing a sports game on a computer, some people tend to have a similar behavior to show happiness or frustration about the course of the game. In a live sporting event like a horse racing, people believe their behavior can influence the course of the race. To build 
a game that simulates this reality, we need to detect the user behavior and to change the gameplay dynamically. This is related to affective gaming [8] which is included in the topic of affective computing [9]. According to Gilleade et al [4], to have an affective videogame, both the player and the game have to be responsive to the affective signals of the other. Many games have been proposed [3] that address the challenge of capturing the emotional behavior of the player and include it in the gameplay. One of the main differences between them is the way they capture the emotional information. Affective approaches are based on, at least, one of the following modalities: detection of the facial expression [1], analysis of the body gestures [6] and motion [10], voice analysis [7] and physiological signal changes [11]. Despite the high number of proposals, the process of detection and interpretation of the emotional information, in order to be used in a game, remains a major challenge [2].

This paper proposes a game that detects changes in the user behavior when playing and use them to change the gameplay of a horse racing game. We developed a prototype of a Web game that detects the motion and the audio produced by the player using a webcam.

We want to explore the user behavior in contexts where the player has an enormous tendency to get excited. At the same time, we want to build sporting games that gives sensations close to the ones we have when we go to the stadium. This proposal does not really interpret the emotions generated by the player. Our proposal is in the middle of a natural user interface (NUI) game [5] and an affective game. Initially, the player acts by naturally moving his body and screaming as a result of his enthusiasm. When the player realizes that he only needs to make noise and move his body to influence the horse performance, the game becomes a NUI game. Gilleade et al [4] discuss this question in some proposals and they called them biofeedback games. To overcome this question we ascribe to the horses different personalities. That is, each horse reacts differently to the user enthusiasm.

The main purpose of this work is to answer the following questions:

- Can we design an affective game without interpreting the real emotion of the player?

- How we put a player that already understand the game mechanics reacting naturally to the game?

This work is a part of a project where the goal is to study and develop a platform to detect affective signals to be used in games. The prototype presented is the output of a study that tries to find answers to the above questions. The project includes the study of a multi-modal approach to be used in affective games.

\section{Game Overview}

The main blocks of the horse racing game are (see Figure 1):

- User Interaction - is used to capture the affective signals of the user when playing. At this stage, it is composed of a webcam to capture the body motion and the audio produced by the player; 


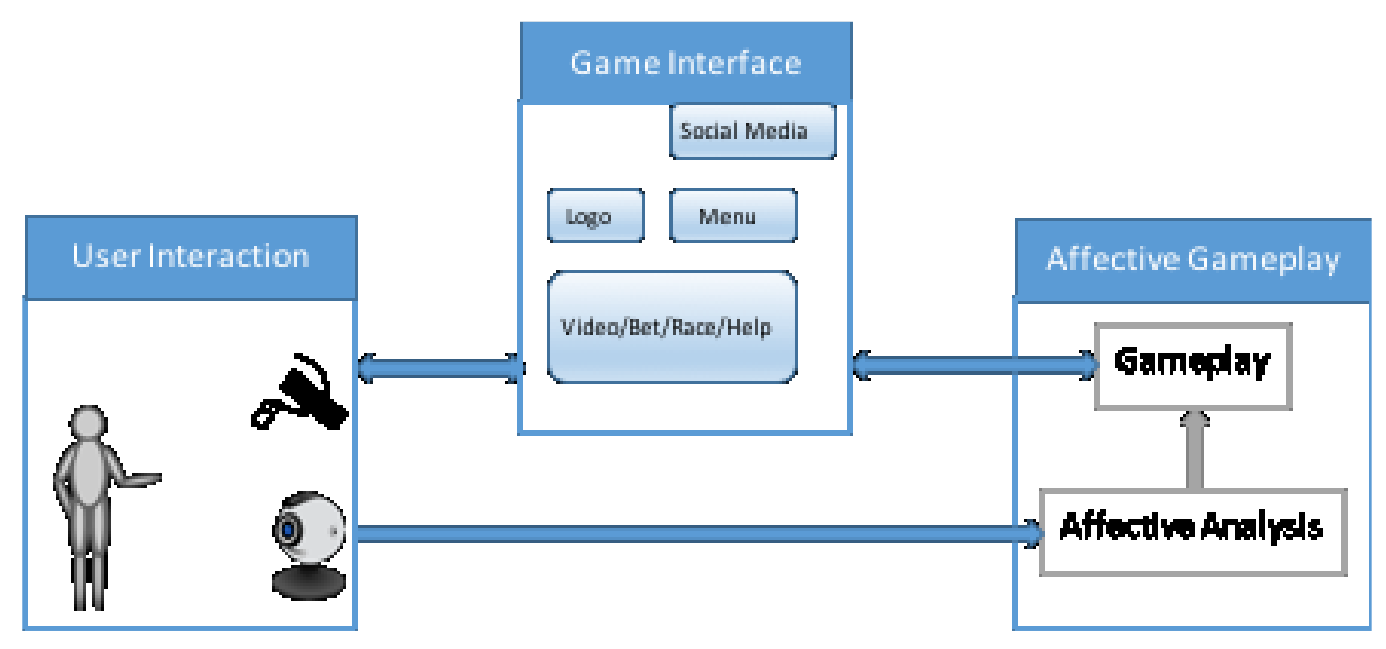

Figure 1. Main blocks of the horse racing game.

- Game Interface - is composed of (see Figure 2) a collection of social media hyperlinks, the logo of the game, a menu and an area to display the different layouts of the game (Video/Bet/Race/Help);

- Affective Gameplay - is divided in two parts, the Gameplay and the Affective Analysis part. The second is used to extract motion and sound from the webcam and to detect if there are some relevant changes in the user behavior (body movement and noise). This information is given to the Gameplay in or order to change the course of the game.

\section{Game Design}

We decided to consider children attending primary school as target audience. Children are more genuine in their emotions and therefore are suitable to our goals. We had to also include teenagers and adults because in some parts of the design process, particularly in issues

related to usability, it was difficult to get relevant (mature) feedback only with children.

After analyzing the target audience, we developed sketches on paper that have been refined progressively by members of our team and by some adults that we included in the audience. Finally, we built a functional Web prototype that were refined based on the output of usability tests performed with the audience. Figures $2,3,4$ and 5 illustrate the main layouts of the user interface.

The goal of the game is to earn money by betting in the winning horse of each race. Figure 3 shows the "Help" layout that describes the main steps of the game loop:

(1) select the option "Play" of the menu, (2) select the horse and make the bet, (3) motivate the horse with 


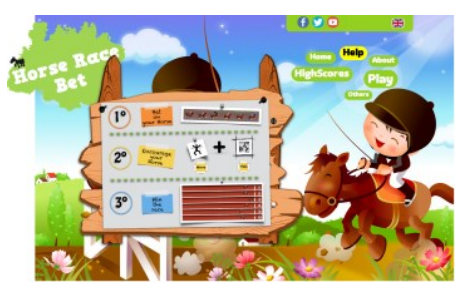

Figure 3: Help layout - this layout presents the three main steps of a move.

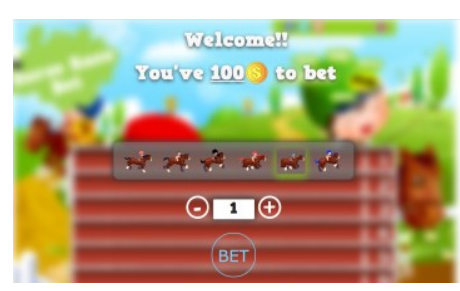

Figure 4: Bet layout - this layout is used to select the horse and to make the bet.

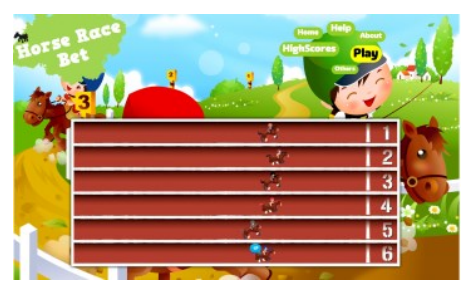

Figure 5: Race layout - this image illustrates the horse race. enthusiasm and (4) win the race. We also have a video explaining the game in the main layout (see figure 2 ).

Some players mentioned during the tests, we should add to the interface elements to give feedback about the detected user enthusiasm. At this stage, we did not follow this suggestion because we want the player be genuine in his emotions. We feel this can contribute to promote a controlled user behavior.

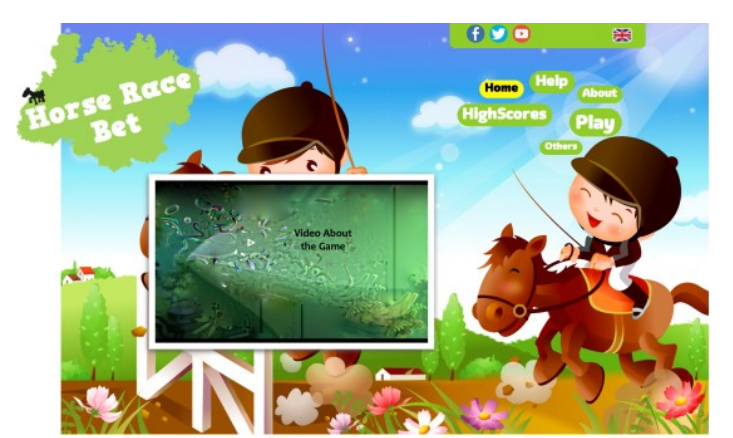

Figure 2: Horse Racing Game - Main Layout.

\section{Affective Analysis}

The audio signal is analyzed by calculating the energy of the captured sound in time windows of 185 milliseconds. This value was chosen based on experiences with users. The energy value obtained is normalized (value between 0 and 1 ) and used to quantify the emotion level of the player, according to the decision intervals presented in Figure 6 . The values of the limits $\mathrm{T}(\mathrm{T} 0, \ldots, \mathrm{T} 4$ in Figure 6$)$ are obtained removing the noise of the environment (in our experience, the values used are, $0,0.1,0.25,0.55$ e 1 , respectively). The video is analyzed by computing the number of active pixels of the image obtained by the subtraction of two video frames. Active pixels are the pixels which the difference are greater than a threshold (we used the value of 40 in our experiments). For subtraction, the frames selected are synchronized with the beginning and the end of the time window used to analyze the audio signal. Thus, the audio and video used are related to the same period of time. The number of the active pixels obtained is used to quantify the emotion level, similarly to the audio (using the decision intervals in Figure 6). For the video signal, the values of the limits $\mathrm{T}(\mathrm{TO}, \ldots, \mathrm{T} 4)$, used in our experience are, 0, 8, 15, 30 e 100, respectively. The values are conditioned by the illumination of the environment. For both signals, we calculate four levels to quantify the user behavior (see Figure 6):

- Level 0 - no signs of any emotion/enthusiasm;

- Level 1 - significant movement of the body (e.g., arms movement) or power sound enough so that the player may have said anything in a low tone. Both signals are relevant to be considered as small emotional reaction;

- Level 2 - high movement (e.g., movement of the arms, body and head) or high power, the player can have screamed or spoken with great intensity. Relevant to be considered as an emotional reaction without doubts;

- Level 3 - too much movement or too high power, not considered as an emotion. Perhaps the player has realized how the game works and starts to make a lot of noise near the microphone.

The limits of the intervals were selected based on the motion and the sound produced during the playability and usability tests (the same strategy used to select the size of the time window). 


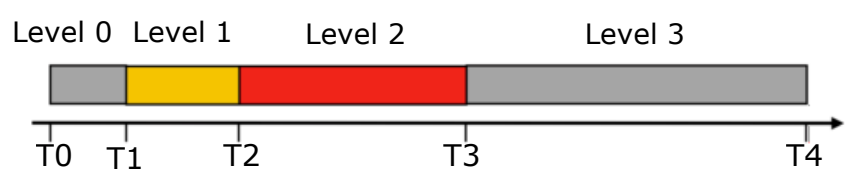

Figure 6: Audio and video: decision intervals to quantify the user behavior/enthusiasm.

\section{Gameplay}

After selecting the option "Play" in the menu, the player begins by choosing one of the horses available and the number of coins to bet. The number of coins must be greater than 0 and can not exceed the total number of coins available by the player.

To use the webcam in the browser we have to ask the user for permission. The game can be played without the detection of the user behavior/enthusiasm. In this case, all horses have the same attributes (minimum and maximum speed), even the one chosen by the player. The speed of each horse is randomly selected between the maximum and minimum values.

If the chosen horse wins, the player gains twice the bet amount selected. If the horse loses, the player loses all coins used in the bet. The game ends when the player lose all the money.

When the player gives permission to use the webcam, we divided the horses in 4 types of sensibilities:

- Same sensitivity to emotional signals obtained either by audio or by video;
- More sensitive to emotional signals obtained by the audio;

- More sensitive to emotional signals obtained by the video;

- Low sensitivity to emotional signals.

Each horse is characterized by 4 attributes: minimum speed, maximum speed, audio sensibility and video sensibility. The audio and video sensibilities are translated in speed values (according to type of the horse sensibility) and added to the horse speed randomly selected between the maximum and minimum values.

\section{Results and Future Research}

To test the game, we started by estimating the parameters of the audio and video algorithms. We asked 7 users ( 2 children, 2 teenagers and 3 adults) to test our game with the webcam turned off. That is, the emotional signals of the player are not included in the gameplay. After experiencing the game, we asked users to give their opinion. We also used this initial tests to gathered data to estimate the parameters of the algorithms. Following this, we asked the users to do the same tests now guided by a questionnaire. At the end of the questionnaire the users have to answer general questions about the game. First of all, in some cases the behavior of the users was not the same. In future, we need to include more data in order to build more generalized algorithms. Adult and teenager users criticized the user interface for being too childish. Children did not notice the difference between the two sessions. This was a good result for us. We chose children because we think they can help us to answer the second question of our research. Adult and teenager users, from the moment they discovered how 
to win, they change to a more controlled behavior. Sometimes, because of the different personalities of the horses and the last quantification level of their behavior, some users temporarily had some doubts about the game mechanics, but did not return to play naturally. A game essentially needs to be fun. The users give us that feedback but one affective game has to take advantage of the emotions. In the future, we will continue to work on this part through the use of other sensors, namely the Kinect sensor, because it captures 3D information which allow us to improve the detection (and interpretation) of the body movements. Based on the feedback we had, we also plan to continue the study with children and adults in order to compare their behaviors. We have to include more children in our study because we want to have more evidences that children are more emotionally genuine. Concerning the first question, given the difficulty in interpreting the emotions, through the study we performed to make this game, there are evidences that in some applications, it is not necessary to interpret the user emotions.

\section{Acknowledgements}

This work was supported by FCT/MEC NOVA LINCS PEst UID/CEC/04516/2013.

\section{References}

1. I. Bacivarov and P. Corcoran. 2009. Facial expression modeling using component AAM models - Gaming applications. International IEEE Consumer Electronics Society's Games Innovations Conference, 1-16.

2. R. Calvo and S. D'Mello. 2010. Affect Detection: An Interdisciplinary Review of Models, Methods, and Their Applications. IEEE Transactions on Affective Computing 1, 1: 18-37.
3. T. Christy and L. Kuncheva. 2014. Technological Advancements in Affective Gaming: A Historical Survey. GSTF International Journal on Computing (JoC) 3, 4: 32-41.

4. K. Gilleade, A. Dix, and J. Allanson. 2005. Affective Videogames and Modes of Affective Gaming: Assist Me, Challenge Me, Emote Me. Proceeding of DIGRA Conference on Changing Views: Worlds in Play 8: 547554.

5. G. Glonek and M. Pietruszka. 2012. Natural User Interfaces. Journal of Applied Computer Science 20, 2: 27-45.

6. L. Grace. 2013. Big huggin: a bear for affection gaming. CHI'13 Extended Abstracts on Human Factors in Computing Systems, 2919-2922.

7. C. Jones and A. Deeming. 2008. Affective humanrobotic interaction. Lecture Notes in Computer Science 4868 LNCS: $175-185$.

8. I. Kotsia, S. Zafeiriou, and S. Fotopoulos. 2013. Affective gaming: A comprehensive survey. IEEE Computer Society Conference on Computer Vision and Pattern Recognition Workshops: 663-670.

9. R. Picard. 2000. Affective Computing, The MIT Press.

10. J. Sanghvi, G. Castellano, I. Leite, A. Pereira, P. McOwan, and A. Paiva. 2011. Automatic analysis of affective postures and body motion to detect engagement with a game companion. Proceedings of the 6th International Conference on Human-robot Interaction, 305-312.

11. C. Stockhausen, J. Smyzek, and D. Krömker. 2013. Beats down: Using heart rate for game interaction in mobile settings. Lecture Notes in Computer Science 8120 LNCS: $523-530$. 